\title{
Exact and Quantum Chemistry-like calculations in Helium doped clusters: The $\mathrm{He}_{2} \mathrm{Br}_{2}(X)$ example
}

\author{
O. Roncero ${ }^{1}$, R. Pérez de Tudela, M. P. de Lara-Castells, \\ R. Prosmiti, G. Delgado-Barrio, and P. Villarreal \\ Instituto de Matemáticas y Física Fundamental (C.S.I.C.), \\ Serrano 123, E-28006-Madrid, Spain
}

\begin{abstract}
A quantum chemistry-like approach has been recently developed in our group to deal with $\mathrm{He}_{N}-\mathrm{BC}$ doped helium clusters, where the $\mathrm{BC}$ dopant is a conventional diatomic molecule. The central idea is to consider the He atoms as "electrons" while the $\mathrm{B}$ and $\mathrm{C}$ atoms play the role of the nuclei in standard electronic structure calculations. The procedure provides energies and wavefunctions allowing to perform spectral simulations and, hence, making feasible to do proper comparisons with current experiments. However, due to the large difference of masses of He and electrons, and also to the replacement of Coulomb potentials by molecular interactions, it is worthy to assess to what extent the approximations involved in this model (decoupling of orbital angular momenta of the He atoms from the $\mathrm{BC}$ rotation and adiabatic separation of the $\mathrm{BC}$ stretch versus the He motions) lead to accurate results. In this work we address these issues on the ${ }^{4} \mathrm{He}_{2}-\mathrm{Br}_{2}(X)$ system, containing a couple of bosonic He atoms for which variational calculations can be performed.
\end{abstract}

\footnotetext{
${ }^{1}$ Author to whom correspondence should be addressed. E-mail: oroncero@imaff.cfmac.csic.es
} 


\section{INTRODUCTION}

The unusual features found in spectroscopic experiments on molecules immersed in helium clusters have raised challenging issues related to the quantum nature of this environment. ${ }^{1}$ Following the pioneering work on the infrared spectra of $\mathrm{SF}_{6}{ }^{2,3}$ and $\mathrm{OCS}^{4,5}$ embedded in He droplets, further rotationally resolved spectra have been reported for an ample variety of systems. ${ }^{6-8}$ More recently high-resolution infrared and microwave measurements on small doped helium clusters have been carried out. ${ }^{9,10}$ In turn, several theoretical approaches have been used to describe the structure and ground-state energetics of helium clusters as, e.g., zero temperature Diffusion Monte Carlo and finite temperature Feynman Path-Integral Monte Carlo methods. ${ }^{11}$

Recently, we have developed an alternative quantum chemistry-like approach to obtain energies and structural properties of $\mathrm{He}_{N}-\mathrm{BC}(X)$ complexes. ${ }^{12-15}$ One appealing feature of this treatment is that it supplies system's wavefunctions which allow to carry out spectral simulations. There are, however, two approximations involved which deserve to be carefully analyzed: (a) Decoupling of the orbital angular momentum of the He atoms from the diatomic rotation, and (b) Adiabaticity with respect to the diatomic vibration. In this paper we study the ${ }^{4} \mathrm{He}_{N}-\mathrm{Br}_{2}, N=2$ cluster, a sort of system amenable to perform variational calculations, ${ }^{16-22}$ and for which extensions up to $N=5$ have been recently carried out. ${ }^{23}$ We firstly address issue (a) by comparing methods which couple/uncouple the $\mathrm{BC}$ rotation to the He atoms orbital angular momentum (frozing the BC bond length), designated as adiabatic/quantum chemistry-like methods. Then, issue (b) is tackled by comparing quantum chemistry, adiabatic and diabatic results for selected states.

The paper is organized as follows. In the next section we present a brief outline of the theoretical model describing the Hamiltonian, the symmetry-adapted basis set functions, and the different approaches we use. In Section III, we characterize the 
model potential energy surface (PES) employed, provide the details of the numerical calculations and present the results obtained. The summary and the outlook are outlined in Section IV.

\section{THEORETICAL MODEL}

\section{A. Hamiltonian and Wave Functions}

Using satellite coordinates $\left\{\left(\mathbf{r}, \mathbf{R}_{k}\right)\right\}$, where $\mathbf{r}$ is the vector joining the $\mathrm{B}$ and $\mathrm{C}$ atoms and $\mathbf{R}_{k}$ are the vectors from the center of mass of the $\mathrm{BC}$ molecule to the different He atoms, one can write the Hamiltonian of the $\mathrm{He}_{2}-\mathrm{BC}$ system as ${ }^{16}$

$$
H=H^{\mathrm{d}}+\sum_{k=1}^{2} H_{k}^{\mathrm{t}}\left(\mathbf{R}_{k}, r\right)+\tilde{V}_{12}
$$

consisting of a diatomic part which corresponds to the BC molecule

$$
H^{\mathrm{d}}=-\frac{\hbar^{2}}{2 m} \frac{\partial^{2}}{\partial r^{2}}+U(r)+\frac{\mathbf{j}^{2}}{2 m r^{2}}
$$

plus the He-BC triatomic Hamiltonians, $H_{k}^{\mathrm{t}},(k=1,2)$, and the He-He interaction $\tilde{V}_{12}$ which includes a potential term $V_{12}$ and also a kinetic energy coupling arising from the use of non-Jacobi coordinates,

$$
\tilde{V}_{12}=V_{12}\left(\left|\mathbf{R}_{k}-\mathbf{R}_{l}\right|\right)-\frac{\hbar^{2}}{m_{B}+m_{C}} \nabla_{1} \cdot \nabla_{2}
$$

In Eq. (2), $m$ is the reduced mass of the diatomic molecule, $\mathbf{j}$ is the angular momentum associated with $\mathbf{r}$, and $U$ represents the intramolecular diatomic potential. The triatomic Hamiltonians have the form

$$
H_{k}^{\mathrm{t}}\left(\mathbf{R}_{k}, r\right)=-\frac{\hbar^{2}}{2 \mu} \frac{\partial^{2}}{\partial R_{k}^{2}}+\frac{\mathbf{l}_{k}^{2}}{2 \mu R_{k}^{2}}+W\left(R_{k}, r, \theta_{k}\right)
$$


where $\mu$ is the reduced mass of the $\mathrm{He}-\mathrm{BC}$ system, $\mathbf{l}_{k}$ is the angular momentum associated with $\mathbf{R}_{k}$, and $W$ represents the atom-diatom intermolecular potential that depends on the pair of $\left(R_{k}, r\right)$ distances and the angle $\theta_{k}$ between the $\mathbf{R}_{k}$ and $\mathbf{r}$ vectors.

We choose a body-fixed $(\mathrm{BF})$ coordinate system with the $Z^{\mathrm{BF}}$ axis parallel to $\mathbf{r}$, and denote by $\lambda_{k}$ the quantum numbers associated with the projections of $\mathbf{l}_{k}, k=1,2$, on $Z^{\mathrm{BF}}$. The total wave function is constructed as a linear combination of functions of the form

$$
\Psi_{v q_{1} q_{2}}^{J M \Omega}=\chi_{v}(r) \Phi_{\Omega q_{1} q_{2}}^{J M}\left(\hat{\mathbf{r}}, \mathbf{R}_{1}, \mathbf{R}_{2}\right)
$$

where $\chi_{v}$ is a vibrational state of the (non-rotating) diatomic subunit

$$
\left[-\frac{\hbar^{2}}{2 m} \frac{\partial^{2}}{\partial r^{2}}+U(r)-\epsilon_{v}\right] \chi_{v}(r)=0 .
$$

In Eq. (5),

$$
\Phi_{\Omega q_{1} q_{2}}^{J M}\left(\hat{\mathbf{r}}, \mathbf{R}_{1}, \mathbf{R}_{2}\right)=\mathcal{D}_{M \Omega}^{J^{*}}\left(\varphi_{r}, \theta_{r}, 0\right) \psi_{q_{1} q_{2}}\left(\mathbf{R}_{1}, \mathbf{R}_{2}\right)
$$

where the $\mathcal{D}_{M \Omega}^{J}$ functions are normalized Wigner rotation matrices that depend on the polar components of $\mathbf{r}$ in the space-fixed (SF) frame, $J$ is the quantum number associated with the total angular momentum $\mathbf{J}=\mathbf{j}+\mathbf{l}_{1}+\mathbf{l}_{2}$, while $M$ and $\Omega\left(=\lambda_{1}+\lambda_{2}\right)$ are quantum numbers associated with the projections of $\mathbf{J}$ on $Z^{\mathrm{SF}}$ and $Z^{\mathrm{BF}}$, respectively. In Eq. (7) the $\psi_{q_{1} q_{2}}$ functions are

$$
\psi_{q_{1} q_{2}}\left(\mathbf{R}_{1}, \mathbf{R}_{2}\right)=\prod_{i=1}^{2} f_{n_{i}}\left(R_{i}\right) Y_{\ell_{i} \lambda_{i}}\left(\hat{\mathbf{R}}_{i}\right)
$$

where $q_{i}=\left\{n_{i} \ell_{i} \lambda_{i}\right\}, i=1,2$, denote a set of quantum numbers necessary to specify the relative motion of each He atom with respect to BC.In Eq. (8) the $f_{n_{i}}$ radial functions are associated with He-BC stretching motions and $Y_{\ell_{i} \lambda_{i}}$ are spherical harmonics.

The symmetry operations of the system are the total inversion $\mathcal{E}^{*}$, the permutation of the He atoms, $\mathcal{P}_{12}$, and, if BC is homonuclear, the exchange of diatomic nuclei, $\mathcal{P}_{\mathrm{BC}}$. 
The action of these operators over the functions of Eq.(5) is

$$
\mathcal{E}^{*}\left[\Psi_{v q_{1} q_{2}}^{J M \Omega}\right]=(-1)^{J} \Psi_{v \tilde{q}_{1} \tilde{q}_{2}}^{J M-\Omega}
$$

where $\tilde{q}_{i}=\left\{n_{i} \ell_{i}-\lambda_{i}\right\}$

$$
\mathcal{P}_{12}\left[\Psi_{v q_{1} q_{2}}^{J M \Omega}\right]=\Psi_{v q_{2} q_{1}}^{J M \Omega}
$$

and

$$
\mathcal{P}_{\mathrm{BC}}\left[\Psi_{v q_{1} q_{2}}^{J M \Omega}\right]=(-1)^{J+l_{1}+l_{2}} \Psi_{v \tilde{q}_{1} \tilde{q}_{2}}^{J M-\Omega}
$$

Hence, a symmetry-adapted basis set is constructed ${ }^{18}$ from the functions of Eq.(5) as

$$
\Psi_{v q_{1} q_{2}}^{J M \Omega \kappa \eta}=\mathcal{N}\left\{\left[\Psi_{v q_{1} q_{2}}^{J M \Omega}+\kappa \Psi_{v q_{2} q_{1}}^{J M \Omega}\right]+\varepsilon(-1)^{J}\left[\Psi_{v \tilde{q}_{1} \tilde{q}_{2}}^{J M-\Omega}+\kappa \Psi_{v \tilde{q}_{2} \tilde{q}_{1}}^{J M-\Omega}\right]\right\}
$$

which are eigenfunctions of $\mathcal{E}^{*}, \mathcal{P}_{12}$, and $\mathcal{P}_{\mathrm{BC}}$, with eigenvalues $\varepsilon, \kappa$, and $\eta=\varepsilon(-1)^{\ell_{1}+\ell_{2}}$, respectively. In Eq. (12), $\Omega \geq 0$, and $\mathcal{N}$ is a normalization factor.

\section{B. Diabatic, adiabatic, and quantum chemistry-like treatments}

In an exact treatment, the wave function is expanded in terms of the basis functions of Eq.(12) where the corresponding sum runs over quantum numbers $v, q_{1}, q_{2}, \Omega$ up to convergence.

Taking advantage of the mismatch existing between the low frequency of the He$\mathrm{BC}$ stretching/bending motions and the high frequency of the $\mathrm{BC}$ vibrations, one can consider just a unique diatomic vibration in the above mentioned expansion and the diabatic wave function is written as

$$
\Psi^{\mathrm{D}} \simeq \chi_{v}(r) \Phi_{J M}^{\mathrm{D}}\left(\mathbf{R}_{1}, \mathbf{R}_{2}, \hat{\mathbf{r}}\right)=\chi_{v}(r) \sum_{\Omega q_{1} q_{2}} c_{\Omega q_{1} q_{2}} \Phi_{\Omega q_{1} q_{2}}^{J M}\left(\hat{\mathbf{r}}, \mathbf{R}_{1}, \mathbf{R}_{2}\right)
$$

where the $c_{\Omega q_{1} q_{2}}$ coefficients, and the corresponding $E_{v J}^{\mathrm{D}}$ eigenvalues, are obtained by solving the Schrödinger equation associated to the effective Hamiltonian

$$
H^{(v)}=-\frac{\hbar^{2}}{2 \mu} \frac{\partial^{2}}{\partial R_{1}^{2}}+\frac{\mathbf{l}_{1}^{2}}{2 \mu R_{1}^{2}}+W_{v}\left(R_{1}, \theta_{1}\right)
$$




$$
\begin{aligned}
& -\frac{\hbar^{2}}{2 \mu} \frac{\partial^{2}}{\partial R_{2}^{2}}+\frac{\mathbf{l}_{2}^{2}}{2 \mu R_{2}^{2}}+W_{v}\left(R_{2}, \theta_{2}\right) \\
& +B_{v} \mathbf{j}^{2}+\tilde{V}_{12}
\end{aligned}
$$

In Eq. (14), the vibrational diatomic function $\chi_{v}$ is used to average the triatomic HeBC potentials $W_{v}\left(R_{k}, \theta_{k}\right)=\left\langle\chi_{v}\left|W\left(R_{k}, r, \theta_{k}\right)\right| \chi_{v}\right\rangle$, and to obtain an effective diatomic rotational constant $B_{v}=\left\langle\chi_{v}\left|r^{-2}\right| \chi_{v}\right\rangle$. This constitutes the diabatic (D) vibrational approach, firstly proposed for triatomic systems ${ }^{24}$ and also applied to tetraatomic van der Waals systems ${ }^{16,18}$ in which one considers that the diatomic vibration remains unchanged within the cluster. Such approach is expected to be accurate for the lower diatomic vibrational states. Note that, within this approach, the total energy of the system is $E_{v J}^{\mathrm{D}}+\epsilon_{v}$.

In the adiabatic $(\mathrm{AD})$ approximation one retains in a first step the rotational term of the diatomic Hamiltonian $H^{\mathrm{d}}$ and solves, for different fixed values of the diatomic bond length $r$,the Schrödinger equation

$$
\left[\sum_{k=1}^{2} H_{k}^{\mathrm{t}}\left(\mathbf{R}_{k} ; r\right)+\frac{\mathbf{j}^{2}}{2 m r^{2}}+\tilde{V}_{12}-E_{J}^{\mathrm{AD}}(r)\right] \Phi_{J M}^{\mathrm{AD}}\left(\mathbf{R}_{1}, \mathbf{R}_{2}, \hat{\mathbf{r}} ; r\right)=0
$$

where $\Phi_{J M}^{\mathrm{AD}}=\sum_{\Omega q_{1} q_{2}} c_{\Omega q_{1} q_{2}}(r) \Phi_{\Omega q_{1} q_{2}}^{J M}\left(\hat{\mathbf{r}}, \mathbf{R}_{1}, \mathbf{R}_{2}\right)$. In Eq. (15), each $r$-dependent eigenenergy $E_{J}^{\mathrm{AD}}(r)$ constitutes an additional potential energy term for the diatomic molecule. Writing down the wave-function as a simple product

$$
\Psi^{\mathrm{AD}} \simeq \chi_{J v}^{\mathrm{AD}}(r) \Phi_{J M}^{\mathrm{AD}}\left(\mathbf{R}_{1}, \mathbf{R}_{2}, \hat{\mathbf{r}} ; r\right)
$$

the $\chi_{J v}^{\mathrm{AD}}$ functions describe the vibrational states of a distorted diatomic molecule which are solutions of the Schrödinger equation

$$
\left[-\frac{\hbar^{2}}{2 m} \frac{\partial^{2}}{\partial r^{2}}+U(r)+E_{J}^{\mathrm{AD}}(r)-\varepsilon_{J v}^{\mathrm{AD}}\right] \chi_{J v}^{\mathrm{AD}}(r)=0
$$

where $v$ is the stretching (vibrational) quantum number. Note that now the total energy of the system becomes $\varepsilon_{J v}^{\mathrm{AD}}$. 
The quantum chemistry (QC) treatment, in turn, starts from the adiabatic approach but completely neglects the diatomic Hamiltonian, i.e., considers BC as fixed "nuclei". So, one solves at fixed $r$ values

$$
\left[\sum_{k=1}^{2} H_{k}^{\mathrm{t}}\left(\mathbf{R}_{k} ; r\right)+\tilde{V}_{12}-E^{\mathrm{QC}}(r)\right] \psi^{\mathrm{QC}}\left(\mathbf{R}_{1}, \mathbf{R}_{2} ; r\right)=0,
$$

looking for just the ground level. Writing again the wave-function as a simple product

$$
\Psi^{\mathrm{QC}} \simeq \chi_{J \Omega v}^{\mathrm{QC}}(r) \mathcal{D}_{M \Omega}^{J^{*}}\left(\varphi_{r}, \theta_{r}, 0\right) \psi^{\mathrm{QC}}\left(\mathbf{R}_{1}, \mathbf{R}_{2} ; r\right)
$$

where $\psi^{\mathrm{QC}}=\sum_{q_{1} q_{2}} c_{q_{1} q_{2}}(r) \psi_{q_{1} q_{2}}\left(\mathbf{R}_{1}, \mathbf{R}_{2}\right)$, one considers a distortion of the diatomic potential that includes, in addition to $E^{\mathrm{QC}}(r)$ of Eq. (18), an average of the diatomic rotation,

$$
\left[-\frac{\hbar^{2}}{2 m} \frac{\partial^{2}}{\partial r^{2}}+U(r)+E^{\mathrm{QC}}(r)+\frac{\left\langle\mathbf{j}^{2}\right\rangle}{2 m r^{2}}-\varepsilon_{J \Omega v}^{Q C}\right] \chi_{J \Omega v}^{\mathrm{QC}}(r)=0,
$$

where, neglecting Coriolis couplings, one has ${ }^{12,13,15}$

$$
\left\langle\mathbf{j}^{2}\right\rangle \approx\left\langle\mathbf{L}^{2}\right\rangle+\hbar^{2}\left[J(J+1)-2 \Omega^{2}\right]
$$

and the $\mathbf{L}^{2}$ average, $\mathbf{L}=\mathbf{l}_{1}+\mathbf{l}_{2}$, is computed by using the distribution of $L$ values ${ }^{14}$ in the $\psi^{\mathrm{QC}}$ state. In Eq.(21), $\Omega$ is a good quantum number already determined at solving Eq.(18). Therefore, in this approach, the total energy of the system is $\varepsilon_{J \Omega v}^{\mathrm{QC}}$.

Concerning with the relative computational cost, the $\mathrm{D}$ and $\mathrm{AD}$ approachs are roughly comparable (the later linearly scales with the number of points accounted for in the adiabatic variable). In turn, the neglect of Coriolis couplings involved in the QC approach reduces significantly the size of the matrices to be diagonalized, which in addition are independent of the total angular momentum $J$ considered. This becomes of crucial importance for $J>0$ states of larger clusters where, in D or AD approachs, the number of basis functions would dramatically increase with the number of helium atoms. 


\section{PES, NUMERICAL DETAILS, AND RESULTS}

\section{A. Potential Energy Surface}

We have shown ${ }^{22}$ that a model potential energy surface (PES) of the entire system can be constructed as a sum of ab initio $\mathrm{He}-\mathrm{Br}_{2}$ triatomic interactions ${ }^{25}$ and semiempirical $^{26} \mathrm{He}-\mathrm{He}$ pair interactions. DMC calculations ${ }^{27}$ have indicated that the energy and density distributions for this cluster with $N=2$ are only weakly dependent on the $\mathrm{He}-\mathrm{Br}_{2}$ interaction, irrespective of whether it is described by the ab initio triatomic potential with minima at T-shaped and linear configurations, ${ }^{25,28}$ or by a sum of $\mathrm{He}-\mathrm{Br}$ and $\mathrm{Br}-\mathrm{Br}$ Morse potentials with a minimum only at the T-shaped configuration. ${ }^{29}$ So, and as in recent works ${ }^{12,13,23}$ we use this last model of PES, i.e., the simple addition of atom-atom interactions. The $\operatorname{Br}_{2}(X)$ intermolecular interaction $U$ was described by a Morse function ${ }^{30}$

$$
U(r)=D\left\{1-\exp \left[-\alpha\left(r-r_{e q}\right)\right]\right\}^{2}
$$

while each $\mathrm{He}_{-} \mathrm{Br}_{2}(X)$ intramolecular potential $W$ was approximated through addition of Morse-type He-Br pair interactions ${ }^{29}$

$$
W(r, s, \theta)=M\left(s_{1}\right)+M\left(s_{2}\right)
$$

where $s_{i}, i=1,2$, are the two He-Br distances and

$$
M\left(s_{i}\right)=D^{\prime}\left\{1-\exp \left[-\alpha\left(s_{i}-s_{e q}\right)\right]\right\}^{2}-D^{\prime}
$$

and the He-He interaction is also described by a Morse potential. ${ }^{31}$ The corresponding parameters can be found at TABLE I of Ref. [14]. However, in the present calculations no truncation was imposed to the He-He interaction. 


\section{B. Numerical Details}

In the calculations presented here the following masses $(a m u)$ were used: $m_{\mathrm{Br}}=$ 78.91830, and $m_{4 \mathrm{He}}=4.00260$.

The radial $f_{n}(R)$ functions appearing at Eq. (8) were obtained as follows. Fixing the $\mathrm{Br}_{2}$ bond length at its equilibrium value, we look for the ground level of the triatomic He-Br ${ }_{2}$ subsystem at different fixed orientations $\theta_{n}$, by solving the Schrödinger equation

$$
\left[-\frac{\hbar^{2}}{2 \mu} \frac{\partial^{2}}{\partial R^{2}}+W\left(R, \theta_{n}, r_{e q}\right)-E_{n}(r)\right] g_{n}\left(R, \theta_{n}\right)=0
$$

the $g_{n}\left(R, \theta_{n}\right)$ functions being then orthogonalized through a Schmidt procedure, which leads to an orthonormal set of $f_{n}(R)$ functions. A grid of 4096 points in the $R$ range of [1.5 - 18.5] Å was employed to numerically solve Eq. (25) using a Numerov procedure. To describe properly the neighborhood of the equilibrium angular region, the chosen set of $\theta_{n}$ values is $\theta_{n}=\pi / 2-(n-1) \pi / 24, n=1, n_{\max }$. Convergence in energies up to $10^{-5} \mathrm{~cm}^{-1}$ was achieved by using $\ell_{\max }=12$, all the possible $\lambda$ values, and $n_{\max }=4$.

The necessary integrals were evaluated numerically in the radial variables and analytically in the angular ones. To this end, 100 angles equally spaced in the intervals $[0, \pi / 2]$ and $[0, \pi]$ were considered for describing the triatomic interactions $W$ appearing at Eq. (4) and the He-He potential $V_{12}$ of Eq. (3), respectively. As usual, these potentials were further expanded in Legendre polynomials. For all the operators involved, the corresponding matrix elements in the present uncoupled representation can be found in Appendix A of Ref [14], with the exception of $\mathbf{j}^{2}$, which can be easily obtained using angular momentum algebra. ${ }^{32}$

\section{Results}

Owing to the boson character of the He atoms, all the calculations are performed imposing an even parity for their exchange, i.e., $\kappa=+1$. The rest of symmetries 
correspond to states of the lowest energy at each value of the total angular momentum $J$ considered, that is, inversion parity and exchange of $\operatorname{Br}$ atoms $\varepsilon=\eta=(-1)^{J}$. For these states, one therefore obtains $\ell_{1}+\ell_{2}=$ even. In QC calculations, the ground level obtained by solving Eq. (18) corresponds to a " $\Sigma$ " state, i.e., $\Omega=0$.

The effect of decoupling the orbital angular momenta of the He atoms and the $\mathrm{BC}$ rotation is analyzed by comparing the results obtained from the $\mathrm{QC}$ and $\mathrm{AD}$ approaches. At three different elongations of the $\mathrm{Br}_{2}$ bond, Table I collects the interaction energies $E^{Q C}(r)$ (column 2), and $E^{Q C}(r)+R$ (column 3), with $R=<\mathbf{j}^{2}>/ 2 m r^{2}$ resulting from Eq. (21) for $J=0$. Also in this table we list the AD energies obtained by solving Eq. (15) for $J=0,1$, and 2 (columns 4 to 6 ). As can be realized, at $J=0$, the QC approach underestimates the binding energies by $\sim 0.1 \mathrm{~cm}^{-1}$ with respect to the $\mathrm{AD}$ values at all the $r$ distances.

The results of the precedent table are then used, after a linear fitting, to obtain the corresponding perturbation to the diatomic potential energy within the $\mathrm{QC}$ and $\mathrm{AD}$ approaches. For different $(J, v)$ states, we list in Table II the QC, AD, and D total energies of the system obtained by solving Eq. (20), Eq. (17), and the Schrödinger equation for the Hamiltonian of Eq. (14), respectively. In order to facilitate comparisons, the corresponding energies for the bare diatomic molecule are listed in the last column of this table. The $\mathrm{D}$ values can be considered as exact with an accuracy of $10^{-3} \mathrm{~cm}^{-1}$ (a full variational calculation including $v=0,1,2$ leads to an energy of $127.00454 \mathrm{~cm}^{-1}$ for the $(0,0)$ state). As a rule, the accord between AD and D results is always better than $0.01 \mathrm{~cm}^{-1}$, although somewhat better for the lowest vibrational state $v=0$, no matter the rotational excitation of the cluster. On the other hand, the difference between $\mathrm{QC}$ and $\mathrm{AD}$ values remains, according to the results shown in Table I, of the order of $0.1 \mathrm{~cm}^{-1}$ irrespective of the vibrational state, but increases with $J$. For instance, at $(J, v)=(2,1)$, the mismatch reaches a value of $\sim 0.2 \mathrm{~cm}^{-1}$. We would like 
to point out that, in spite of this discrepancy, the QC approach predicts the position of the most intense vibrotational $(v=1 \leftarrow 0)$ Raman $_{\text {branch }}^{12,13} \mathrm{Q}(0)$, determined by the difference of energies between the $(0,1)$ and $(0,0)$ states, within an accuracy of $\sim 10^{-3} \mathrm{~cm}^{-1}\left(\sim 10^{-2} \mathrm{~cm}^{-1}\right)$ with respect to the AD (D) prediction. However, for the $\mathrm{S}(0)$ branch, which is $\sim 10$ times less intense than $\mathrm{Q}(0),{ }^{12,13}$ the $\mathrm{QC}$ accuracy is only of $\sim 0.1 \mathrm{~cm}^{-1}$ when compared with $\mathrm{AD}$ or $\mathrm{D}$ approaches.

The differences between the ground vibrational wavefunction $\chi_{0}$ of the bare $\mathrm{Br}_{2}$ molecule and the corresponding adiabatic $\chi_{0}^{\mathrm{AD}}$ and quantum chemistry $\chi_{0}^{\mathrm{QC}}$ functions of the distorted diatomic within the cluster, at $J=0$, are displayed in Figure 1 as functions of $r$. In the two cases, such differences vary in the range of $[-7,7] \times 10^{-4}$ $\AA^{-1 / 2}$, while the difference $\chi_{0}^{\mathrm{QC}}-\chi_{0}^{\mathrm{AD}}$ also shown in that Figure is almost negligible. According to first order perturbation theory, the solutions of Eq. (17) in terms of the solutions of Eq. (6) would approximately be

$$
\chi_{v}^{\mathrm{AD}}-\chi_{v} \approx \sum_{v^{\prime} \neq v} \frac{\left\langle\chi_{v^{\prime}}\left|E^{\mathrm{AD}}\right| \chi_{v}\right\rangle}{\epsilon_{v}-\epsilon_{v^{\prime}}} \chi_{v^{\prime}} .
$$

Therefore, this difference looks like an excited $v=1$ vibrational function, the dominant term in Eq. (26), albeit modulated by the small coefficient $\left\langle\chi_{1}\left|E^{\mathrm{AD}}\right| \chi_{0}\right\rangle /\left(\epsilon_{0}-\epsilon_{1}\right)$.

For the $(J, v)=(0,1)$ state, a similar plot is shown in Figure 2. One can realize that the differences $\chi_{1^{-}} \chi_{1}^{\mathrm{AD}}$ and $\chi_{1^{-}} \chi_{1}^{\mathrm{QC}}$ increase with respect to the ground level case, varying over the interval $[-15,9] \times 10^{-4} \AA^{-1 / 2}$. They look like an excited $v=2$ state since, due to the anharmonicity of the diatomic potential, this term should become dominant in Eq. (26). As can be seen the difference $\chi_{1}^{\mathrm{QC}}-\chi_{1}^{\mathrm{AD}}$ is also somewhat bigger than for the $v=0$ case.

Taking into account the proximity of the different diatomic wave functions, which are relevant for the estimation of matrix elements of induced dipole moments through the polarizability of the molecule, ${ }^{12}$ one can state that the intensity of the Raman $\mathrm{Q}(0)$ branch is well estimated within the $\mathrm{QC}$ or $\mathrm{AD}$ approaches. 


\section{SUMMARY AND OUTLOOK}

The assumptions involved in a quantum chemistry-like treatment of diatomic molecules embedded in helium clusters have been tested on the ${ }^{4} \mathrm{He}_{2}-\mathrm{Br}_{2}(X)$ system using a model potential energy surface. To this end, we performed a study of the energies of the system and wave functions associated to the diatomic subunit within $\mathrm{QC}$ as well as AD and D (exact) treatments. Our main conclusions are:

(1) Adiabaticity of the diatomic stretch is accurate within 0.01 wavenumbers with respect to the diabatic, exact treatment, and constitutes an acceptable approximation.

(2) Decoupling of BC rotation from the He angular momenta is the main source of discrepancies when one compares $\mathrm{QC}$ with $\mathrm{AD}$ or exact results, the accuracy being of $0.1 \mathrm{~cm}^{-1}$ at $J=0$. So, its effect is one order of magnitude more important than the adiabatic assumption, and is magnified as $J$ increases.

The QC treatments can provide qualitative, or even semi-quantitative, descriptions of the main features associated to the spectroscopy of this kind of clusters, although they should be improved in order to provide quantitative predictions. Also, it would be useful to study the accuracy of this approximation in different systems involving faster rotors and/or asymmetric rotors.

Note, however, that the failure of the decoupling among rotational motions comes from the fact that only one state (the ground level of the complex) is involved in the further calculation of the distorting potential, as is the case of Hartree (or Hartree-Fock for fermions) methods already applied. ${ }^{12,13,15}$ However, if one accounts for additional excited states, the QC approach constitutes the first step of a pre-diagonalization procedure which, in principle, would lead to an outstanding increase in accuracy. In this regard, the results obtained with the recently developed Jacobi-Davidson based full interaction configuration method for doped ${ }^{3} \mathrm{He}_{N}$ clusters ${ }^{33}$ are particularly encourag- 
ing. Work is in progress to extend the method to boson and mixtures of boson/fermion environments.

\section{ACKNOWLEDGMENTS}

This work has been partially supported by the DGICYT Spanish grants FIS200402461 and CTQ2004-02415/BQU. M.P.deL.-C. and R.P. acknowledge the support of the Spanish "Ramón y Cajal" Programme, Grant Nos: PDRyC-2003-001015 and PDRyC-2006-001017. The calculations presented here were performed at Centro de Cálculo of IMAFF (CSIC). 


\section{References}

${ }^{1}$ J. P. Toennies and A. F. Vilesov, Angew. Chem., Int. Ed. 43, 2622 (2004).

${ }^{2}$ S. Goyal, D. L. Schutt, and G. Scoles, Phys. Rev. Lett. 69, 933 (1992).

${ }^{3}$ M. Hartmann, R. E. Miller, J. Toennies, and A. F. Vilesov, Phys. Rev. Lett. 75, 1566 (1995).

${ }^{4}$ S. Grebenev, J. P. Toennies, and A. F. Vilesov, Science 279, 2083 (1998).

${ }^{5}$ S. Grebenev, M. Hartmann, M. Havenith, B. Sartakov, J. P. Toennies, and A. F. Vilesov, J. Chem. Phys. 112, 4485 (2000).

${ }^{6}$ K. Nauta and R. E. Miller, J. Chem. Phys. 115, 10138 (2001).

${ }^{7}$ K. Nauta and R. E. Miller, J. Chem. Phys. 117, 4846 (2002).

${ }^{8}$ C. M. Lindsay and R. E. Miller, J. Chem. Phys. 122, 104306 (2005).

${ }^{9}$ J. Tang and A. R. W. McKellar, J. Chem. Phys. 119, 754 (2003).

${ }^{10}$ A. R. W. McKellar, J. Chem. Phys. 121, 6868 (2004).

${ }^{11}$ For a recent review, see M. Barranco, R. Guardiola, S. Hernández, R. Mayol, J. Navarro, and M. Pí, J. Low. Temp. Phys. 142, 1 (2006).

${ }^{12}$ D. López-Durán, M. P. de Lara-Castells, G. Delgado-Barrio, P. Villarreal, C. D. Paola, F. A. Gianturco, and J. Jellinek, Phys. Rev. Lett. 93, 053401 (2004).

${ }^{13}$ D. López-Durán, M. P. de Lara-Castells, G. Delgado-Barrio, P. Villarreal, C. D. Paola, F. A. Gianturco, and J. Jellinek, J. Chem. Phys. 121, 2975 (2004).

${ }^{14}$ M. P. de Lara-Castells, D. López-Durán, G. Delgado-Barrio, P. Villarreal, C. D. Paola, F. A. Gianturco, and J. Jellinek, Phys. Rev. A 71, 033203 (2005). 
${ }^{15}$ M. P. de Lara-Castells, R. Prosmiti, G. Delgado-Barrio, D. López-Durán, P. Villarreal, F. A. Gianturco, and J. Jellinek, Phys. Rev. A 74, 053201 (2006).

${ }^{16}$ P. Villarreal, O. Roncero, and G. Delgado-Barrio, J. Chem. Phys. 101, 2217 (1994).

${ }^{17}$ M. I. Hernández and N. Halberstadt, J. Chem. Phys. 100, 7828 (1994).

${ }^{18}$ M. I. Hernández, N. Halberstadt, W. D. Sands, and K. C. Janda, J. Chem. Phys. 113, $7252(2000)$.

${ }^{19}$ A. Heidenreich, U. Even, and J. Jortner, J. Chem. Phys. 115, 10175 (2001).

${ }^{20}$ A. Heidenreich and J. Jortner, J. Chem. Phys. 118, 10101 (2003).

${ }^{21}$ P. M. Felker and D. Neuhauser, J. Chem. Phys. 119, 5558 (2003).

${ }^{22}$ A. Valdés, R. Prosmiti, P. Villarreal, and G. Delgado-Barrio, J. Chem. Phys. 122, $044305(2005)$.

${ }^{23}$ P. M. Felker, J. Chem. Phys. 125, 184313 (2006).

${ }^{24}$ J. A. Beswick and J. Jortner, Adv. Chem. Phys. 47, 363 (1981).

${ }^{25}$ R. Prosmiti, C. Cunha, P. Villarreal, and G. Delgado-Barrio, J. Chem. Phys. 116, 9249 (2002).

${ }^{26}$ R. A. Aziz and M. J. Slaman, J. Chem. Phys. 94, 8047 (1991).

${ }^{27}$ C. D. Paola, F. A. Gianturco, D. López-Durán, M. P. de Lara-Castells, G. DelgadoBarrio, P. Villarreal, and J. Jellinek, Chem Phys. Chem 6, 1348 (2005).

${ }^{28}$ A. Valdés, R. Prosmiti, P. Villarreal, and G. Delgado-Barrio, Mol. Phys. 102, 2277 (2004). 
${ }^{29}$ T. González-Lezana, M. I. Hernández, G. Delgado-Barrio, A. A. Buchachenko, and P. Villarreal, J. Chem. Phys. 105, 7454 (1996).

${ }^{30}$ K. P. Huber and G. Herzberg, Constants of Diatomic Molecules, Van Nostrand Reinhold, New York, 1979.

${ }^{31}$ Z. Bačić, M. Kennedy-Mandzink, and J. W. Moskowitz, J. Chem. Phys. 105, 6472 (1992).

${ }^{32}$ R. N. Zare, Angular Momentum (Wiley, New York, 1988) .

${ }^{33}$ M. P. de Lara-Castells, G. Delgado-Barrio, P. Villarreal, and A. O. Mitrushchenkov, J. Chem. Phys. 125, 221101 (2006). 
TABLE I. Interaction energies (in $\mathrm{cm}^{-1}$ ) of the $\mathrm{Br}_{2}-\mathrm{He}_{2}$ cluster at three distances of the diatomic bond $r$ (in $\AA$ ). $E^{\mathrm{QC}}$ : from QC calculations; $E^{\mathrm{QC}}+R$ : plus averaged rotational term $(J=0) ; E^{\mathrm{AD}}(J)$ : from $\mathrm{AD}$ calculations at a total angular momentum $J$.

\begin{tabular}{|l|c|c|c|c|c|}
\hline \multicolumn{1}{|c|}{$r$} & $E^{\mathrm{QC}}$ & $E^{\mathrm{QC}}+R$ & $E^{\mathrm{AD}}(0)$ & $E^{\mathrm{AD}}(1)$ & $E^{\mathrm{AD}}(2)$ \\
\hline \hline & & & & & \\
2.20 & -36.50056 & -35.45019 & -35.55011 & -35.40962 & -35.13286 \\
2.35 & -36.30716 & -35.30165 & -35.38971 & -35.25735 & -34.99577 \\
& -36.14551 & -35.17602 & -35.25510 & -35.12913 & -34.87964 \\
\hline
\end{tabular}

TABLE II. Total energies (in $\left.\mathrm{cm}^{-1}\right)$ of the cluster for selected $(J, v)$ states from QC, $\mathrm{AD}$ and $\mathrm{D}$ calculations. The corresponding energies for the bare $\mathrm{Br}_{2}(X)$ molecule are also listed in the last column.

\begin{tabular}{|c|c|c|c|c|}
\hline$(J, v)$ & $\mathrm{QC}$ & $\mathrm{AD}$ & $\mathrm{D}$ & $\mathrm{Br}_{2}(X)$ \\
\hline \hline & & & & \\
$(0,0)$ & 127.08983 & 127.00180 & 127.00491 & 162.38608 \\
$(1,0)$ & 127.25372 & 127.13404 & 127.13704 & 162.54998 \\
$(0,1)$ & 450.25752 & 450.17036 & 450.17823 & 485.54225 \\
$(2,1)$ & 450.74725 & 450.56217 & 450.57028 & 486.03197 \\
& & & & \\
\hline
\end{tabular}




\section{FIGURE CAPTIONS}

FIG. 1. Differences between the $v=0$ vibrational wave function of the isolated diatomic molecule and, at $J=0$, those distorted by the presence of the surrounding He atoms obtained through the adiabatic and quantum chemistry approaches, as well as the difference between the later ones.

FIG. 2. Same as in FIG. 1, but for the vibrational diatomic state $v=1$. 


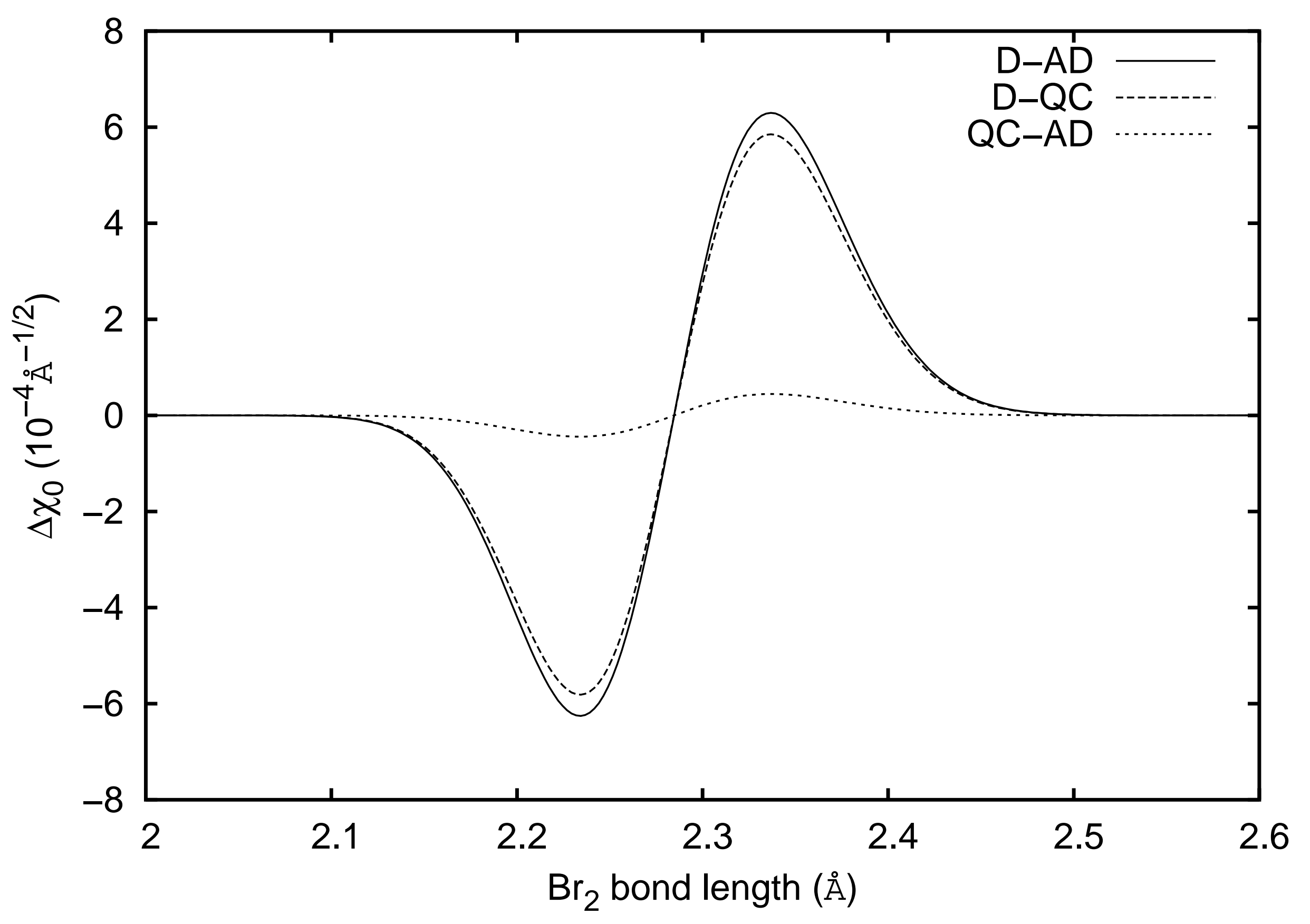




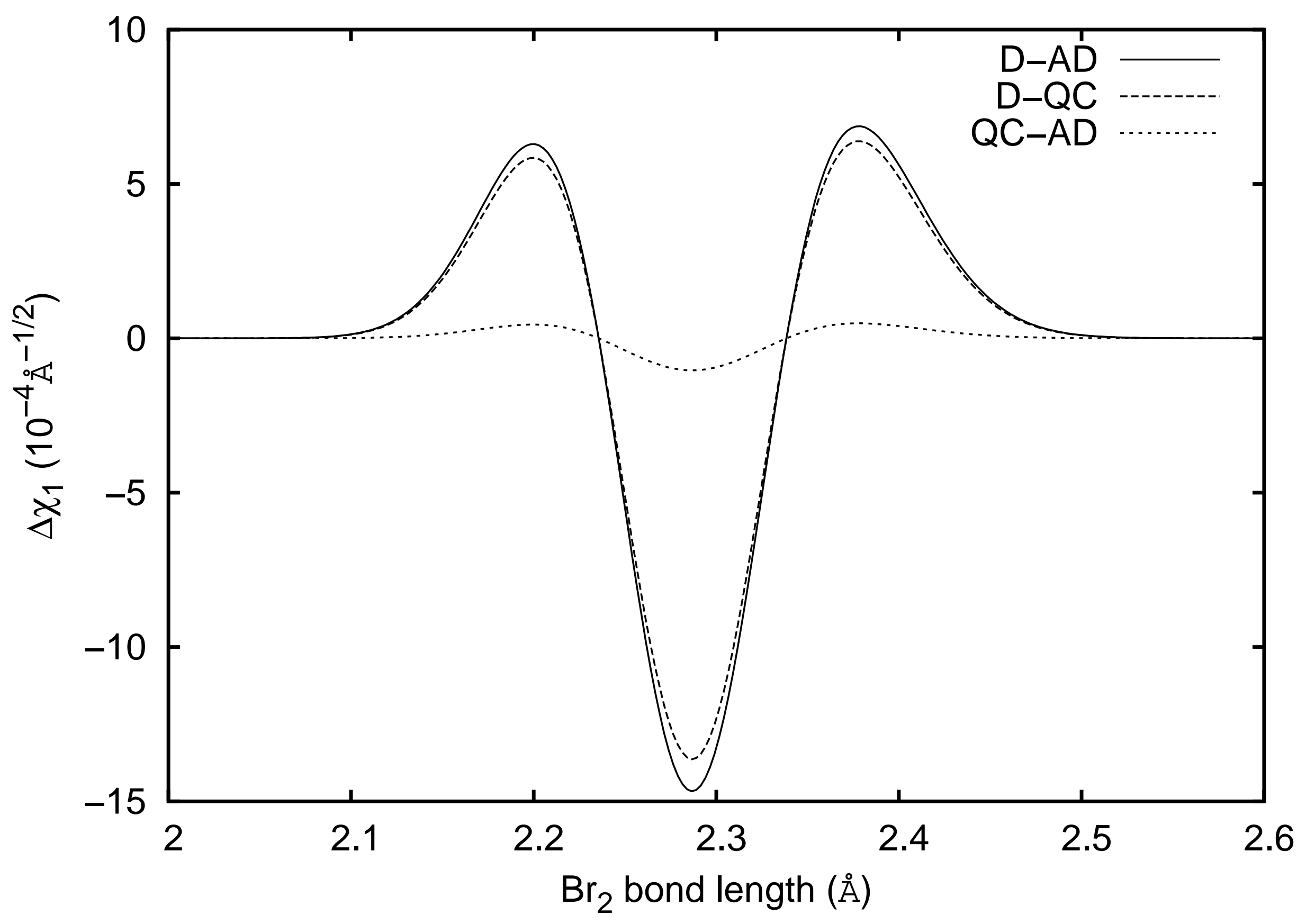

